\title{
Model Predictive Control of Autonomous Underwater Vehicles Based on Horizon Optimization
}

\author{
Sudirman \\ Department of Electrical Engineering \\ Institut Teknologi Sepuluh Nopember \\ (ITS) \\ Surabaya, Indonesia \\ sudirman13@ee.its.ac.id
}

\author{
Mochammad Rameli \\ Department of Electrical Engineering \\ Institut Teknologi Sepuluh Nopember \\ (ITS) \\ Surabaya, Indonesia \\ rameli@ee.its.ac.id
}

\author{
Rusdhianto Effendi \\ Department of Electrical Engineering \\ Institut Teknologi Sepuluh Nopember \\ (ITS) \\ Surabaya, Indonesia \\ rusdhi@elect-eng.its.ac.id
}

\begin{abstract}
The use of Autonomous Underwater Vehicle (AUV) for underwater exploration is increasingly popular because of its superiority compared to its predecessor, the Remote Operated Vehicle (ROV), in terms of its wider range. The cruise range of an AUV depends not only on how large the capacity of the battery is, but also is determined by how efficient the AUV uses energy in completing its mission. Energy optimization by optimizing the control method is an option. MPC was chosen because of its ability to efficiently use energy by optimizing the delta input of its cost function. The test results show that the selection of the horizon with the right value can produce efficient energy use on the steering actuator.
\end{abstract}

Keywords - Autonomous Underwater Vehicle, Model Predictive Control, Horizon Optimization.

\section{INTRODUCTION}

Autonomous Underwater Vehicle (AUV) received significant attention in recent years. The growing need for underwater exploration makes researchers interested in continuing to develop AUV performance. This is because AUV's advantages over its predecessor like ROV that still using the cable so that the cruising range is very limited. While jobs such as seafloor mapping, oil exploration, geological sampling, subsea environments surveillance and defense needs require vehicles that have wider coverage and are able to overcome barriers of unpredictable underwater conditions. With AUV some of these tasks not only can be implemented in a wider range, but also can work without human supervision, this is of course useful especially if the number of available human resources is minimal.

However, the high nonlinearity causes the dynamics of AUV to vary with respect to time. To overcome this, more sophisticated control methods are needed to overcome the nonlinearity. In its development various methods controls have been applied to overcome this nonlinearity problem. There are several types of controls that often used, among others PID, Sliding Mode Controller, Fuzzy Neural Network and Model Predictive Control (MPC) or also known as Receding Horizon Control. One of the most frequently used methods is due to its ability to overcome high order nonlinearity [1].

In addition to control issues, energy efficiency issues are also received serious attention from researchers. Energy is one of the main determining factor of AUV's endurance every time it is launched. More operational duration mean more data that can be obtained when conducting underwater exploration. Besides that, duration of the operation will save launch costs, because the process of charging or replacing battery of AUV requires a lots of effort and cost [2].

Therefore we need a variety of possible solutions for increase operational duration of AUV. Increased operational duration can be done by adding battery capacity, refill with nature energy and make energy savings. In [3], Daniel Steinberg et. al. try to uncover whether buoyancy-driven modes of propulsion are intrinsically more or less efficient at maximizing achievable horizontal range than propellerdriven modes of propulsion. Energy Storage Optimization is carried out by Griffiths, et. al. [4], while Teong-Beng and Mandar Chitre [5] succeeded in minimizing energy use with energy-efficient path planning by utilizing ocean current maps.

Meanwhile, MPC is one of the optimal control methods whose results are closest to the Global Optimum Value as produced by Global Optimized Controller [7]. MPC as a controller has reported its success in [11].

In [7], Razaei et al. managed to control the energy consumption of a hybrid car by using energy efficiently. In the same article he found that the selection of the number of horizons on the MPC algorithm greatly affect the optimal value generated. In other words, on a relatively similar trajectory and environment, we can find a horizon value that can be considered as an "optimal horizon".

Therefore in this paper the authors propose the use of MPC for heading control on AUV with optimal horizon control search offline. This is become a challenge due to AUV's nonlinearity.

Using the right number of horizons will also prevent excessive energy consumption while calculating long MPC algorithms. Because it is shown that in the horizon versus efficiency graph, the MPC horizon will be saturated, so the addition of the horizon after certain point will no longer contributes to the efficiency improvement.

This paper is organized as follows. Section 2 presents the problem formulation includes AUV model, MPC algorithm and Efficiency Energy formulation. Control system design are presented in section 3. Simulations result of the proposed 
JAREE-Journal on Advance Research in Electrical Engineering Volume4, Number 1, April 2020

method are presented in section 4. Section 5 present a conclusion of this paper.

\section{PROBLEM FORMULATION}

\section{A. AUV Model}

There are two coordinate that needed in AUV motion: earth-fixed coordinates and body-fixed coordinates (see fig.1). Reference to the earth (earth-fixed coordinates) is a coordinate system used to indicate the position of the AUV towards the center of the earth's axis. While the reference to the body (body-fixed coordinates) is a coordinate system for determining speed and acceleration from AUV which has a center point at the center of gravity or the center of buoyancy

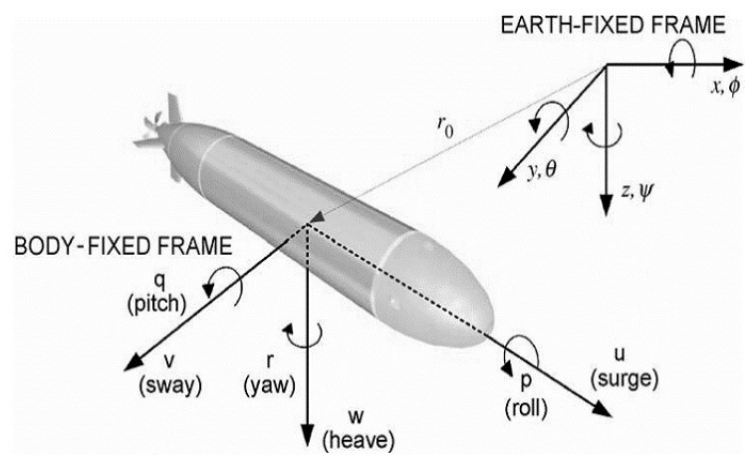

Fig. 1. Coordinate system of AUV

AUV has six DOF (Degree of Freedom) in its motion. Three of them are translation and the three others are rotations of the earth's axis $(\mathrm{x}, \mathrm{y}, \mathrm{z})$. Table 1 show the notations used for each DOF.

\begin{tabular}{|c|c|c|c|c|}
\hline DOF & & $\begin{array}{l}\text { Forces/ } \\
\text { moments }\end{array}$ & $\begin{array}{l}\text { Linear/ } \\
\text { angular }\end{array}$ & $\begin{array}{c}\text { Position / } \\
\text { Euler angles }\end{array}$ \\
\hline 1 & Surge & $X$ & $u$ & $x$ \\
\hline 2 & Sway & $Y$ & $v$ & $\mathrm{y}$ \\
\hline 3 & Heave & $Z$ & $w$ & Z \\
\hline 4 & Roll & $K$ & $p$ & $\phi$ \\
\hline 5 & Pitch & $M$ & $q$ & $\theta$ \\
\hline 6 & Yaw & $N$ & $r$ & $\psi$ \\
\hline
\end{tabular}

Where in vectorial form can be written as:

$$
\begin{array}{rlrl}
\eta & =\left[\eta_{1}^{T}, \eta_{2}^{T}\right]^{T} & r_{11}=[x, y, z]^{T} & \eta_{2}=[\phi, \theta, \Psi]^{T} \\
v=\left[v_{1}^{T}, v_{2}^{T}\right]^{T} & v_{1}=[u, v, w]^{T} & v_{1}=[p, q, H]^{T} \\
\tau=\left[\tau_{1}^{T}, \tau_{2}^{T}\right]^{T} & \tau_{1}=[X, Y, z]^{T} & \tau_{2}=[K, M, \mathrm{~N}]^{T}
\end{array}
$$

where : $\eta$ : position and orientation vector with fix reference to earth (eart-fixed reference).

$v$ : linear and angular velocity vector with fix reference to body (body-fixed reference).

$\tau$ : moment and force vector with fix reference to body

(body-fixed frame).

Below is Equation of Motion of AUV expressed in statespace form:

$$
\left[\begin{array}{cccccc}
a_{11} & 0 & 0 & 0 & a_{15} & a_{16} \\
0 & a_{22} & 0 & a_{24} & 0 & a_{26} \\
0 & 0 & a_{31} & a_{34} & a_{35} & 0 \\
0 & a_{42} & a_{43} & a_{44} & 0 & 0 \\
a_{51} & 0 & a_{51} & 0 & a_{55} & 0 \\
a_{61} & a_{62} & 0 & 0 & 0 & a_{66}
\end{array}\right]\left[\begin{array}{c}
u \\
\dot{v} \\
\dot{v} \\
\dot{w} \\
\dot{v} \\
\dot{q} \\
\dot{w}
\end{array}\right]-
$$

$$
\left[\begin{array}{cccccc}
b_{11} & b_{12} & b_{13} & 0 & b_{15} & 0 \\
0 & b_{21} & 0 & b_{24} & b_{25} & a_{26} \\
0 & 0 & b_{33} & b_{34} & b_{35} & b_{36} \\
0 & 0 & 0 & 0 & 0 & 0 \\
0 & 0 & b_{53} & b_{34} & b_{55} & b_{56} \\
0 & b_{62} & 0 & b_{64} & b_{65} & b a_{66}
\end{array}\right]\left[\begin{array}{c}
u \\
v \\
w \\
p \\
q \\
c
\end{array}\right]=\left[\begin{array}{c}
c 1 \\
c 2 \\
c 3 \\
c 4 \\
c 5 \\
c 6
\end{array}\right]
$$

$B$. where the parameter for each component are :

$$
\begin{aligned}
& a_{11}=(m-X \dot{u}) \quad a_{62}=-\left(m x_{G}-N \dot{v}\right) \quad b_{54}=\left(M_{v p} v+M_{r p} r\right) \\
& a_{15}=m z_{G} \quad a_{66}=\left(I_{z z}-N \dot{r}\right) \quad b_{55}=M_{u q} u \\
& a_{16}=-m y_{G} \quad b_{11}=X_{u|u|}|u| \quad b_{56}=\left(M_{v p} v+M_{r p} r\right) \\
& a_{22}=(m-Y \dot{v}) \quad b_{12}=X_{u v} u \quad b_{62}=N_{u v} u \\
& a_{24}=-m z_{G} \quad b_{13}=\left(X_{u w} u+X_{w|w|}|w|\right) \quad b_{64}=N_{w p} w \\
& a_{35}=-\left(Z \dot{q}+m x_{G}\right) \quad b_{15}=\left(X_{w q} w+X_{q|q|}|q|\right) \quad b_{65}=N_{p q} p \\
& +X_{q q} q \\
& a_{42}=m z_{G} \quad b_{22}=\left(Y_{u v} u+Y_{v|v|}|v|\right) \quad b_{66}=N_{w p} w \\
& a_{43}=-m y_{G} \quad b_{24}=Y_{w p} w \quad c_{1}=X_{H S}+X_{\text {prop }} \\
& a_{44}=\left(I_{x x}-K \dot{p}\right) \quad b_{25}=Y_{p q} p \quad c_{2}=Y_{H S}+Y_{u u \delta_{r}} u^{2}\left(\delta_{r_{\text {top }}}+\delta_{r_{\text {bott }}}\right. \\
& a_{51}=-m z_{G} \quad b_{26}=Y_{w p} w \quad c_{3}=Z_{H S}+Z_{u u \delta_{s}} u^{2} \\
& a_{53}=\left(m x_{G}-M \dot{w}\right) \quad b_{33}=\left(Z_{w|w|}|w|+Z_{u w} u\right) \quad\left(\delta_{s_{\text {right }}}+\delta_{s_{\text {left }}}\right) \\
& a_{55}=\left(I_{y y}-M \dot{q}\right) \quad b_{34}=\left(Z_{v p} v+Z_{r p} r\right) \quad c_{4}=K_{H S}+K_{\text {prop }}+K_{u u \delta_{r}} \\
& \left(\delta_{r_{\text {top }}}+\delta_{r_{\text {bottom }}}\right) \\
& +K_{u u \delta_{s}}\left(\delta_{s_{\text {right }}}+\delta_{s_{\text {left }}}\right) \\
& a_{61}=m y_{G} \quad b_{53}=M_{u w} u \quad c_{5}=M_{H S}+M_{u u \delta_{s}} u^{2} \delta_{s} \\
& c_{6}=N_{H S}+N_{u u \delta_{r}} u^{2} \delta_{r}
\end{aligned}
$$

C.

\section{Model Predictive Control}

To understand the concept of MPC as controller, the system model is given in the discrete time representation as follows:

$$
\begin{aligned}
x_{m}(k+1) & =A_{m} x_{m}(k)+B_{m} u(k) \\
y(k) & =C_{m} x_{m}(k)
\end{aligned}
$$


JAREE-Journal on Advance Research in Electrical Engineering Volume4, Number 1, April 2020

$$
C A^{N_{p}-N_{c}} B \Delta u\left(k_{i}+N_{e}-1\right)
$$

Where $y(k), u(k)$ and $x_{m}(k)$ represent system outputs, inputs and states respectively.

Augmented Model below change control input from $\mathrm{u}(\mathrm{k})$ to $\Delta \mathrm{u}(\mathrm{k})$ :

$$
\begin{aligned}
& \frac{x[k+1)}{\left[\begin{array}{c}
\Delta x_{m}(k+1) \\
y(k+1)
\end{array}\right]}=\overbrace{\left[\begin{array}{cc}
A_{m} & O_{m}^{x} \\
C_{m} A_{m} & 1
\end{array}\right]\left[\begin{array}{l}
\Delta x_{m} \\
y(k)
\end{array}\right]}^{A(k)}+\stackrel{\left.\begin{array}{c}
B_{m} \\
C_{m} B_{m}
\end{array}\right]}{B} \\
& y(k) \quad=\overbrace{O_{m}}^{c} 11]\left[\begin{array}{c}
\Delta x_{m}(k) \\
y(k)
\end{array}\right]
\end{aligned}
$$

where

$$
O_{m}=\overbrace{\left[\begin{array}{llll}
0 & 0 & \ldots & 0
\end{array}\right] .}^{n_{1}}
$$

The next stage in designing a predictive control system is to calculate the predicted plant output with the upcoming control signal as an adjustable variable. This prediction is described in the optimization window. By observing that instant sampling $k_{i}, k_{i}>0$, state vector $x\left(k_{i}\right)$ available through measurement, state $x\left(k_{i}\right)$ provide current plant information. Upcoming control path are notated by $\Delta u\left(k_{i}\right), \Delta u\left(k_{i}+1\right), \ldots u\left(k_{i}+N_{c}+1\right)$, where $N_{c}$ i control horizon. With the given information $x\left(k_{\mathrm{f}}\right)$, the future state variable predicted for $\mathbb{N}_{v}$ step further, where $N_{v}$ known as prediction horizon or optimation window.

With given $x\left(k_{i}\right)$ we can predict $x\left(k_{\mathrm{i}}+1 \mid k_{\mathrm{i}}\right), x\left(k_{\mathrm{i}}+2 \mid k_{\mathrm{i}}\right), \ldots, x\left(k_{\mathrm{i}}+m \mid k_{\mathrm{i}}\right), \ldots x\left(k_{\mathrm{i}}+N_{p} \mid k_{\mathrm{i}}\right)$ where $x\left(k_{i}+n \mid k_{i j}\right)$ is predicted state variable at $k_{i}+m$.

Based on the augmented state space model A, B, C, future state variables are calculated sequentially using the upcoming set of control parameters of the predicted state variable.

$$
\begin{aligned}
x\left(k_{i}+1 \mid k_{i}\right)= & A x\left(k_{i}\right)+B \Delta u\left(k_{i}\right) \\
x\left(k_{i}+2 \mid k_{i}\right)= & A x\left(k_{i}+1 \mid k_{i}\right)+B \Delta u\left(k_{i}+1\right) \\
= & A^{2} x\left(k_{i}\right)+A B \Delta u\left(k_{i}\right)+B \Delta u\left(k_{i}+1\right) \\
x\left(k_{i}+N_{v} \mid k_{i}\right)= & A^{N_{p}} x\left(k_{i}\right)+A^{N_{p}-1} B \Delta u\left(k_{i}\right)+ \\
& A^{N_{p}-2} B \Delta u\left(k_{i}+k_{i}+1\right)+\cdots+ \\
& A^{N_{p}-N_{c}} B \Delta u\left(k_{i}+N_{e}-1\right)
\end{aligned}
$$

Where the predicted output variable is:

$$
\begin{aligned}
& y\left(k_{i}+1 \mid k_{i}\right)= C A x\left(k_{i}\right)+C B \Delta u\left(k_{i}\right) \\
& y\left(k_{i}+2 \mid k_{i}\right)= C A^{2} x\left(k_{i}\right)+C A B \Delta u\left(k_{i}\right)+ \\
& C B \Delta u\left(k_{i}+1\right) \\
& \vdots \\
& y\left(k_{i}+N_{v} \mid k_{i}\right)=C A^{N_{j}} x\left(k_{i}\right)+C A^{N_{p}-1} B \Delta u\left(k_{i}\right)+ \\
& C A^{N_{p}-2} B \Delta u\left(k_{i}+1\right)+\ldots+
\end{aligned}
$$

by defining vectors:

$$
\begin{gathered}
Y=\left[\begin{array}{lllll}
y\left(k_{i}+1 \mid k_{i}\right) & y\left(k_{i}+2 \mid k_{i}\right) & \ldots & y\left(k_{i}+N_{p} \mid k_{i}\right)
\end{array}\right]^{T} \\
\Delta U=\left[\begin{array}{llllll}
\Delta u\left(k_{i}\right) & \Delta u\left(k_{i}+1\right) & \ldots & \Delta u\left(k_{i}+N_{C}+1\right)
\end{array}\right]
\end{gathered}
$$

by changing above equation into the vector form, then

$$
Y=F x\left(k_{i}\right)+\Phi \Delta U
$$

where

$$
\begin{aligned}
F & =\left[\begin{array}{c}
C A \\
C A^{2} \\
\vdots \\
C A^{N_{P}}
\end{array}\right] ; \\
\Phi & =\left[\begin{array}{cccc}
C B & 0 & \ldots & 0 \\
C A B & C B & \cdots & 0 \\
\vdots & \vdots & \ddots & \vdots \\
C A^{X_{p}-1} R & C A^{N_{P}-2} R & \ldots & C A^{N_{P}-N_{L}}
\end{array}\right]
\end{aligned}
$$

For the set point signal given $r\left(k_{i}\right)$ during sampling $k_{i}$, in a prediction horizon, the objective of the control horizon system is to bring the predicted output as close as possible to the signal set point. Assuming that vector data that containing set point information is :

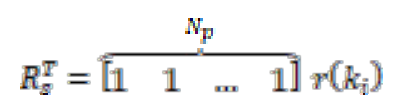

and defines the cost function $\mathrm{J}$ that reflects the control objective as:

$$
I=\left(R_{s}-T^{T}\right)^{T}\left(R_{s}-W\right)+\Delta U^{T} \bar{R} \Delta U
$$

by substituting equation (9) to equation (12) and derived from delta $\mathrm{U}$ equal to zero, we will get the optimal solution control equation as in equation (13) with set point $r\left(k_{i}\right)$

$$
\Delta U=\left(\Phi^{T} \Phi+R\right)^{-1} \Phi^{T}\left(\bar{R}_{\Omega} r\left(k_{i}\right)-F x\left(k_{i}\right)\right)
$$

Although the optimal vector parameter $\Delta U$ contains $\Delta u\left(k_{i}\right), \Delta u\left(k_{i}+1\right), \ldots, \Delta u\left(k_{i}+N_{e}+1\right)$, with the principle of receding horizon control, only applies to the first sampling in each order. So the value of $\Delta u\left(k_{i}\right)$ is

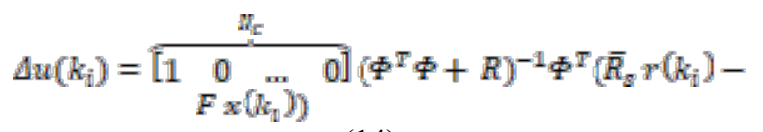

\section{E. Energy Efficiency}

Linear model is used to estimate the required energy for navigating along a known trajectory. This is given as an ordered list of the waypoints described by a 6 DOF vector in 
JAREE-Journal on Advance Research in Electrical Engineering Volume4, Number 1, April 2020

north-east-depth-roll-pitch-yaw (NEDRPY) reference system. The trajectory is then analyzed piecewise, considering pairs of consecutive waypoints as the basic input

for the energy model. Given a pair of waypoints $w A$ and $w B$ in

NEDRPY reference. Using the vehicle attitude at the initial waypoint

According to Carolis [11], the total energy consumption required by AUV to travel through a predetermined trajectory can be illustrated by the following vector:

$$
\mathrm{D}_{\mathrm{AB}}=\left[\begin{array}{llllll}
\Delta_{\mathrm{x}} & \Delta_{\mathrm{y}} & \Delta_{\mathrm{z}} & \Delta_{\varphi} & \Delta_{\mathrm{\theta}} & \Delta_{\psi}
\end{array}\right]^{\mathrm{T}}
$$

Because the depth, roll angle and pitch angle have been controlled to be stable at a certain constant value, $\Delta \mathrm{z}, \Delta \varphi$ and $\Delta \theta$ are zero or do not vary, then the estimated energy needed by AUV to move from one point $\left(w_{A}\right)$ to next point $\left(w_{B}\right)$ is as follow :

$$
\Delta_{\mathrm{E}}\left(\mathrm{w}_{\mathrm{A}}, \mathrm{w}_{\mathrm{B}}\right)=\mathrm{k}_{1} \Delta_{\mathrm{x}}+\mathrm{k}_{2} \Delta_{Y}+\mathrm{k}_{\mathrm{g}} \Delta_{\mathrm{W}}
$$

Because $\Delta \mathrm{x}$ and $\Delta \mathrm{y}$ are proportional to the AUV speed that has been made constant, the effectiveness of the steering controller in conducting energy efficiency can be measured from the total deviation of the yaw or psi angle: :

$$
\mathrm{T}_{\text {energy }}-\sum_{\mathrm{i}=1}^{\mathrm{N}} \Delta_{\Psi}\left(\mathrm{w}_{\mathrm{i}-1}, \mathrm{w}_{\mathrm{i}}\right)
$$

where

$$
\hat{i}=0,1_{x \ldots y} \mathrm{~N}
$$

\section{CONTROL SYSTEM DESIGN}

In order for steering control, we need to control speed, roll angle and depth of AUV. Speed control is done by setting the speed at a value of $1.5 \mathrm{~m} / \mathrm{s}$. Roll angle control is useful for keeping the roll angle at a value of 0 degrees because the roll angle deviation will make it difficult to control the steering, while controlling the depth and controlling the angle of the pitch will keep the AUV at a certain depth (not diving) when controlling the steering.

Block diagram of controller design can be seen in the image below:

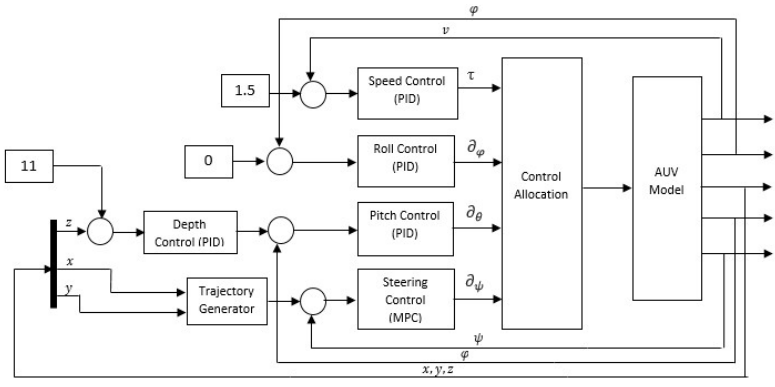

Fig. 2. Control system strategy

\section{A. Linearization}

After the roll condition is maintained at zero degree angle and the depth of the AUV is maintained at 11 meters then steering control is carried out using MPC control.

The MPC design begins by selecting the state that is related to the steering control. Based on an analysis of the AUV dynamics equation, the three states that have the most significant role in steering control are selected, namely the yaw angular velocity (r), the yaw angle $(\psi)$ and the linear velocity in the $y$-axis direction or sway motion $(\mathrm{v})$.

Following are the equations for the three states above and their linearization processes:

$\mathrm{X}$-axis translation $\left(\boldsymbol{F}_{1}\right)$ :

$$
\begin{aligned}
& m \dot{u}-m y_{G} \dot{r}+m z_{G} \dot{q}-X_{\dot{u}} \tilde{u}= \\
& m v r-m w q+m x_{G}\left(q^{2}+r^{2}\right)-m y_{G} p q-m z_{G} p r+ \\
& X_{Y S}+X_{w|u|}|u| u\left|+X_{w V} w v+X_{w W} w w+X_{V|v|}\right| v \mid+ \\
& X_{z} v r+X_{w|w|} w|w|+X_{w a} w q+X_{a a} q q+X_{r r} r T+X_{v r o v}
\end{aligned}
$$

Rotation around Z-axis $\left(\boldsymbol{F}_{2}\right)$ :

$$
\begin{aligned}
& I_{z z} \dot{r}-y_{G} \dot{w}+m x_{G} \dot{v}-N_{v} \dot{v}-N_{r} \dot{r}= \\
& -\left(I_{w v}-I_{x x}\right) p q+m x_{G} w p-m x_{G} w r-y_{G} v^{w}+y_{G} w p+
\end{aligned}
$$

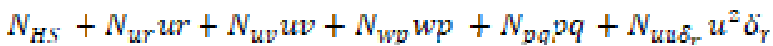

Yaw angle orientation $(\psi)\left(\boldsymbol{F}_{3}\right)$ :

$\psi=\frac{\sin \phi}{\operatorname{mos} A} q+\frac{\cos \phi}{\operatorname{mos} A} r$

Below is linearization matrix of the three equations above:

$$
P X=G X+H U
$$

$$
\left[\begin{array}{ccc}
-x_{\tilde{u}} & -m y_{a} & 0 \\
0 & I_{z z}-N_{\varphi} & 0 \\
0 & 0 & 1
\end{array}\right]\left[\begin{array}{l}
u \\
\dot{r} \\
\dot{\psi}
\end{array}\right]=
$$

$$
\left[\begin{array}{ccc}
d_{11} & d_{11} & 0 \\
d_{11} & d_{11} & 0 \\
0 & d_{11} & 0
\end{array}\right]\left[\begin{array}{l}
u \\
\boldsymbol{r} \\
\psi
\end{array}\right]+\left[\begin{array}{cc}
1 & 0 \\
0 & N_{1 \mu \mathcal{R}_{r}} u^{2} \\
0 & 0
\end{array}\right]\left[\begin{array}{c}
X_{p r o p} \\
\delta_{y}
\end{array}\right]
$$

where

$$
\begin{aligned}
& d_{11}=X_{L|w|}|u|+X_{w w} w \\
& d_{12}=m v+2 m x_{G} T^{r}-m E_{G} p+X_{\mathrm{DT}} v+2 X_{T T}{ }^{2}
\end{aligned}
$$

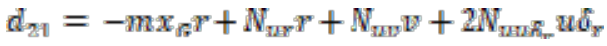

$$
\begin{aligned}
& d_{E Q}--m x_{G} u-y_{G} v+N_{W V} u \\
& d_{32}=\frac{\cos \phi}{\min A}
\end{aligned}
$$


JAREE-Journal on Advance Research in Electrical Engineering Volume4, Number 1, April 2020

Because the AUV speed is made constant, the influence of state ' $u$ ' can be ignored so that the above equation can be written as:

$$
\begin{aligned}
& {\left[\begin{array}{cc}
I_{z z}-N_{\phi} & 0 \\
0 & 1
\end{array}\right]\left[\begin{array}{l}
\dot{\boldsymbol{r}} \\
\dot{\psi}
\end{array}\right]=} \\
& {\left[\begin{array}{cc}
-m x_{G} u-y_{G} v+N_{u \psi} u & 0 \\
\frac{\cos \phi}{\operatorname{mon} \hat{n}} & 0
\end{array}\right]\left[\begin{array}{c}
r_{\psi} \\
\psi
\end{array}\right]+} \\
& {\left[\begin{array}{cc}
0 & N_{u w \varepsilon_{\mathrm{r}}} u^{2} \\
0 & 0
\end{array}\right]\left[\begin{array}{c}
X_{p r o p} \\
\delta_{\tau}
\end{array}\right]}
\end{aligned}
$$

\section{B. Roll and Depth Control}

Before steering control design, roll angle and depth of AUV need stabilized using PID first.

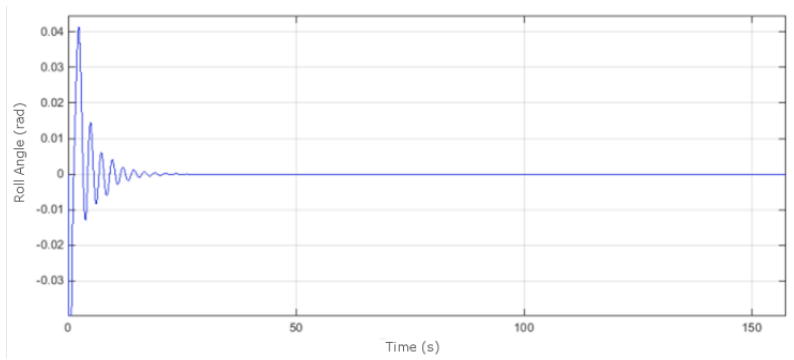

Fig. 3. Roll angle control using PID

From the fig. 3 it can be seen that the roll angle is initially isolated and starts to stabilize at 25 seconds. Isolation that occurs at a maximum of 0.04 radians is a tolerable value.

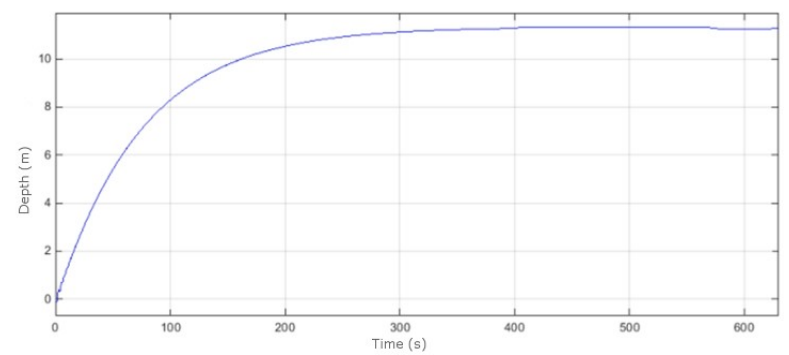

Fig. 4. Depth control using PID

From the fig. 4 it can be seen that AUV continues to dive until it reaches a depth of 11 meters in the 400th second. This can also be seen in relation to the pitch angle in the fig.5, where the pitch angle starts to stabilize around 400 seconds, while the oscillations in the beginning is a roll oscillation effect, this can be easily seen because the oscillation stops almost simultaneously with the cessation of the oscillation on the roll in the 25 th second

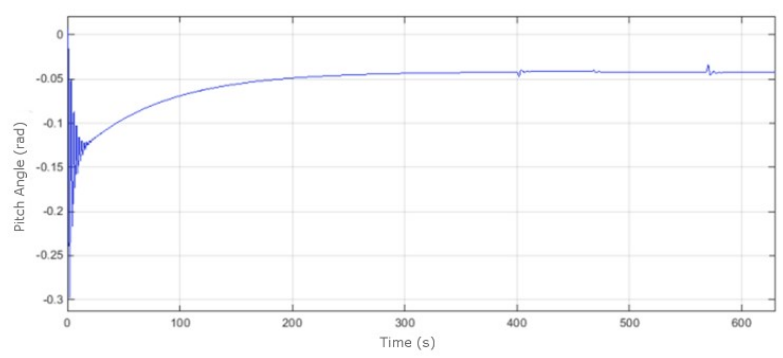

Fig. 5. Pitch control using PID

Based on the results above, we can conclude that the stability of roll angle using PID can achieved in a relatively short time, but the depth was stable in 11 meters after 400 seconds since simulation started

\section{RESULT AND ANALYSIS}

Simulation results show that the proposed method is able to control an AUV to track reference trajectories accurately. To see the effect of the horizon on energy consumption through the total deviation of the psi angle, we will first simulate the value of Rbar $=10$ (fixed) and vary the value of the horizon and illustrate it in the graph.

After the optimal horizon value is obtained, it will be continued by looking at the effect of Rbar on the use of AUV energy by making the value of the horizon constant and making the Rbar value varied.

After simulating by varying the horizon, it is obtained:

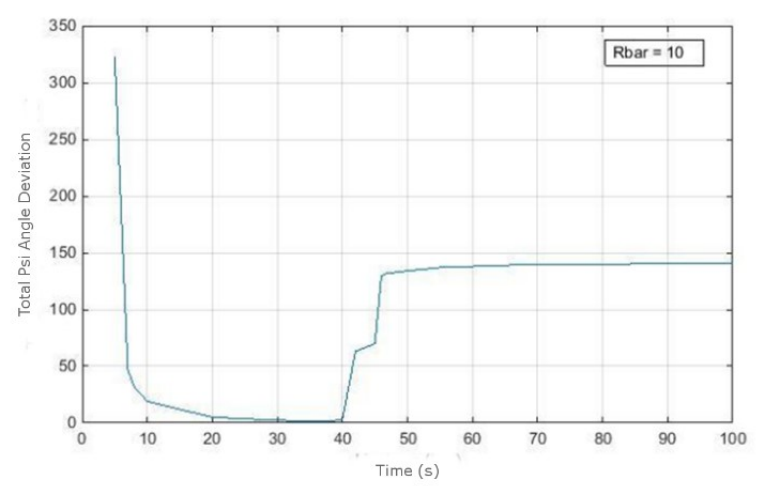

Fig. 6. Effect of Predicted Horizon on energy consumption

From the fig. 6 above we can see that the Optimal Energy Consumption Value is obtained when the Horizon value is 37 , while the addition of the horizon to infinite does not make energy efficiency towards the optimum global value, this is due to the linearization results which have limited accuracy while the MPC method is in dire need high level of accuracy.

In addition to the horizon, one of the parameters that needs to be monitored for its effect on efficient use of energy is Rbar. 
JAREE-Journal on Advance Research in Electrical Engineering Volume4, Number 1, April 2020

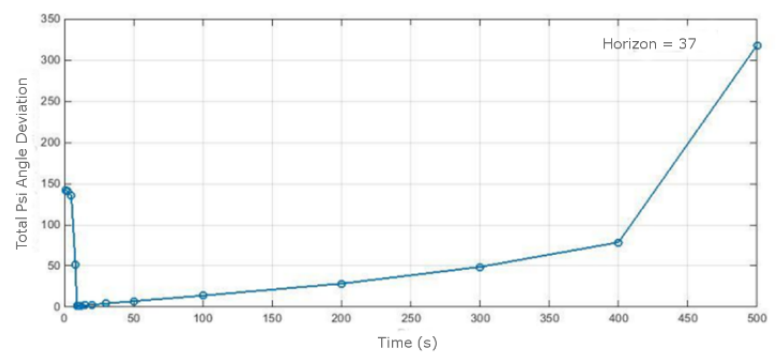

Fig. 7. Effect of Rbar variable on energy consumption

By setting the horizon at 37 and varying the Rbar value, it is found that the optimal Rbar value is 11 .

While the total amount of rudder and fin angles (angular deviations from zero) reaches the lowest point at $\mathrm{Rbar}=100$ which is 3.7. However, with the value of Rbar $=100$, the total value of psi angle deviation is 14.1. This means that at $\mathrm{Rbar}=100$ the energy used to drive the rudder and fin is the least but there is a deviation from a large enough trajectory, meaning that there is a trade-off that we must consider.

Because of the above problem, we decides to choose Rbar $=20$, where the total rudder and fin angles are 3.9 (the difference only 0.2 ) of the total rudder and fin angle deviations, while the total deviation of the resulting psi angle is quite small which is 3 , much smaller than the total rudder and fin angles produced from $\mathrm{Rbar}=100$.

\section{V.CONCLUSION}

From the test results above it is known that the effect of the horizon on the total energy use of the actuator is nonlinear, so the addition of the horizon to infinity or with a very large value on the MPC does not produce Global Optimized Value.

Horizon optimization that has been done, proven to provide a significant energy saving effect. Nevertheless, determination of the optimal horizon and Rbar values in the case of AUV which has high nonlinearity is only effective if applied to AUV control using MPC with a monotonous and repetitive trajectory.

\section{REFERENCES}

[6] Yan Z., Siu F.c. dan Jun W. (2012),"Underwater Vehicles Based on the Simplified Dual Neural Network". IEEE International Conference on Systems, Man, and Cybernetics, 14-17 Oktober 2012, COEX, Seoul, Korea.

[7] Daniel Steinberg, et.al. (2011)," Analysis of Propulsion Methods for Long-Range AUVs". Issues in Technology Theory, Research, and Application. ScholarlyEditions. ${ }^{T M}$, Atlanta, Georgia

[8] G Griffiths, et. al. (2008), "Energy Storage for Long Endurance AUVs", Publication at National Oceanography Centre.

[9] Teong-Beng Koay and Mandar Chitre (2013), "Energy-efficient path planning for fully propelled Energy-efficient Path Planning for Fully Propelled", Conference Publications at OCEANS - Bergen, MTS/IEEE.

[10] Petar Kormushev dan Darwin G. Caldwell (2013), "Improving the Energy Efficiency of Autonomous Underwater Vehicles by Learning to Model Disturbances", Intelligent Robots and Systems (IROS), IEEE/RSJ International Conference, 3-7 November 2013, Tokyo Japan.

[11] Ting Gao, et. al. (2012), "Optimum Design of an AUV by Using Computational Fluid Dynamic Analysis", Proceedings of 2012 International Conference on Mechanical Engineering and Material Science (MEMS 2012)

[12] Amir Rezaei dan Jeffrey B. Burl (2015)," Effects of Time Horizon on Model Predictive Control for Hybrid Electric Vehicles ". IFACPapersOnLine 48-15 (2015) 252-256.

[13] Peter Ridley, Julien Fontan dan Peter Corke (2003),"Submarine Dynamic Modeling". Australian Conference on Robotics \& Automation, Brisbane, Australia.

[14] Peter Ridley, Julien Fontan dan Peter Corke (2003),"Submarine Automatic Control". Proceedings of the Australian Conference on Robotics \& Automation, Brisbane, Australia.

[15] Louis Andrew Gonzalez (2004), "Design, Modelling And Control Of An Autonomous Underwater Vehicle". Thesis at School of Electrical, Electronic and Computer Engineering, The University of Western Australia.

[16] Thor I. Fossen (1994),"Guidance and Control of Ocean Vehicles". John Wiley \& Sons, Ltd., West Sussex, England.

[17] Chen Yang (2007),"Modular Modelling and Control for Autonomous Underwater Vehicle (AUV)". Thesis at Department of Mechanical Engineering, National University of Singapore.

[18] Wang Luiping (2009),"Model Predictive Control System Design and Implementation Using MATLAB". Springer-Verlag London Limited.

[19] Agus Budiyono (2011), "Model Predictive Control for Autonomous Underwater Vehicle". Indian Journal of Geo-Marine Science. Vol. 40(2), April 2011, pp. 191-199.

[20] Valerio De Carolis, David M. Lane, Keith E. Brown (2014)," Lowcost Energy Measurement and Estimation for Autonomous Underwater Vehicles", Proceedings of IEEE-MTS Oceans, Taipei, Taiwan. 